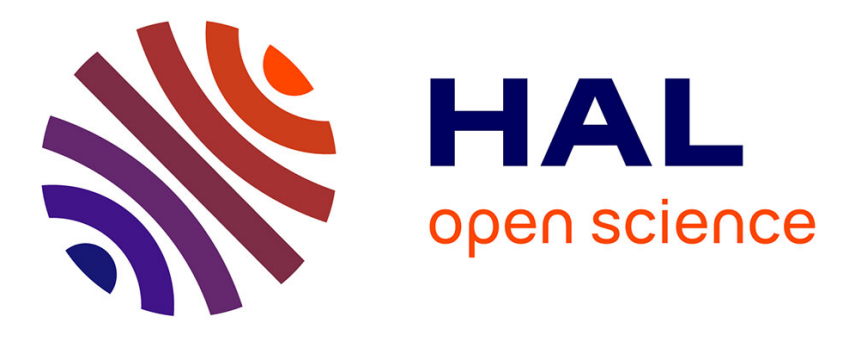

\title{
Plastic and dissipated work and stored energy
}

Olivier Maisonneuve, André Chrysochoos, G. Martin, H. Caumon, J.C. Chezeaux

\section{To cite this version:}

Olivier Maisonneuve, André Chrysochoos, G. Martin, H. Caumon, J.C. Chezeaux. Plastic and dissipated work and stored energy. Nuclear Engineering and Design, 1989, 114 (3), pp.323-333. 10.1016/0029-5493(89)90110-6 . hal-03338960

\section{HAL Id: hal-03338960 https://hal.science/hal-03338960}

Submitted on 9 Sep 2021

HAL is a multi-disciplinary open access archive for the deposit and dissemination of scientific research documents, whether they are published or not. The documents may come from teaching and research institutions in France or abroad, or from public or private research centers.
L'archive ouverte pluridisciplinaire HAL, est destinée au dépôt et à la diffusion de documents scientifiques de niveau recherche, publiés ou non, émanant des établissements d'enseignement et de recherche français ou étrangers, des laboratoires publics ou privés. 


\title{
PLASTIC AND DISSIPATED WORK AND STORED ENERGY
}

\author{
A. CHRYSOCHOOS ${ }^{1}$, O. MAISONNEUVE ${ }^{1}$, G. MARTIN ${ }^{1}$, H. CAUMON $^{2}$ \\ and J.C. CHEZEAUX ${ }^{2}$ \\ ${ }^{1}$ Laboratoire de Mécanique Générale des Milieux Continus, Université Montpellier II, Place Eugène Bataillon, \\ 34060 Montpellier Cédex, France \\ ${ }^{2}$ Laboratoire de Mécanique et d'Acoustique, CNRS-31, chemin Joseph Aiguier, BP 71, 13277 Marseille Cédex, France
}

\begin{abstract}
This paper depicts one theoretical and experimental method to take into account the energy phenomena, associated with the elasto-plastic deformation process, during the elaboration of behaviour laws. The energy balance definition is examined in order to relate the stored energy of cold work to the hardening state variables. Two experimental approaches are used to study the evolution of the energy balance. The first one uses microcalorimetric technique and the second infrared technique. Several industrial metallic materials are studied by both approaches. Energy data are used to control the validity domain of the classical behaviour laws and to elaborate new more appropriate ones. Therefore, the hardening parameters cannot be identified with the thermodynamical forces. The use of energy considerations allows the definition of new thermodynamical forces and state variables, in the case of isotropic or kinematical hardening.
\end{abstract}

\section{Introduction}

This paper describes in a more detailed way, the experimental and theoretical results recently presented in Chrysochoos et al. [1].

When a solid material is strained, its temperature field nearly always changes. The thermal effects, accompanying the deformation process, have been being observed for a long time by the experimentalists (Kelvin [2], Taylor and Quinney [3]), and still remain the subject of a large number of publications (Bui [4], Mandel [5], Krempl [6], etc).

In the case of quasi-static solicitations, the thermal effects are hard to study experimentally because the temperature variations are limited. And this limitation will be so much more important as the strain rate is small and the heat capacity and the heat conduction are large, which is nearly always the case for metallic materials. For instance, with one duralumin strained at $10^{-4} \mathrm{~s}^{-1}$, the temperature variations do not exeed $10^{\circ} \mathrm{K}$.

This could be the reason why thermomechanical couplings are rarely taken into account in the determination of behaviour laws, even if thermodynamics is omnipresent in the construction of models. Generally, the restriction imposed upon the behaviour law is that it verifies the second principle of thermody- namics (using, for instance, the generalized standard materials theory [7]).

It is of great practical interest, and of fundamental importance, to examine this whether the usual laws (the ones integrated in computation codes) are able to describe the energy phenomena associated with deformation processes or not; in the particular case of elastoplastic materials, the theory concerning dissipated energy, or internal energy stored during the hardening, could be in agreement with the experimental observations.

This article depicts, with more technical details than in Chrysochoos et al. [1], the thermomechanical behaviour of metallic elasoplastic materials at finite strain. Three materials, which are commonplace, have been chosen: duralumin (supercooling), carbon steel (agehardening), and stainless steel (age-hardening). An experimental and theoretical methodology is introduced, which first examines the thermomechanical validity of the behaviour laws, and then suggests modifications to produce new and more appropriate laws.

In the first stage, we will restate the definition of the energy balance in the case of elastoplastic materials, during a tensile test at finite strain. Then, two experimental set-ups are described. They make it possible to study the evolution of the energy balance for tensile tests. The first one is based on micro-calorimetric tech- 
niques and allows a global study of the energy phenomena; the second one, using infrared techniques, allows more localized observations.

The results that are obtained are used to control the validity of two classical, nevertheless commonplace models: Prandtl-Reuss's model, based on isotropical hardening, and Prager's model, based on linear kinematical hardening.

Deep disagreement, from an energy point of view, between theoretical and experimental results leads us to define two new laws, which can predict the observed evolution of the energy balance, and be integrated into the Generalized Standard Materials theory. In each case, the energy data are used to define the internal state variable associated with strain hardening and its evolution equation, which is similar to the one of nonlinear kinematical hardening models.

\section{Energy balance}

In the interpretation of the heat quantity measurements used in the energy balance, the experimentalist must be mindful of the several options available in the thermodynamics domain as in the continuous media mechanics domain.

Here, one adopts the classical theory of Irreversible Process Thermodynamics [8,9].

From a mechanical point of view, the dissipation form has been studied for several choices of elastic plastic decomposition in the general case, and then in the case of tensile test $[10,25]$. Some of them are based on propositions made by Green and Naghdi [11], Lee [12] and Nemat-Nasser [13]. We will not consider this in detail here, but it has led us to verify that in the case of tensile test, the dissipation and energy balance forms can be made independent of the most classical kinematical approaches, if the elastic strain remains small.

The curve shown in fig. 1 is characteristic of a 'load-unload cycle' for elastoplastic material and determines the thermodynamical process $(O, A, B)$. The mechanical energy, provided during loading to deform the sample, $W_{\text {ext }}$, can be decomposed into an elastic part, $W_{\mathrm{e}}$, and a complementary part, $W_{\mathrm{a}}$, that we will call 'anelastic' energy

$W_{\text {ext }}=W_{\mathrm{e}}+W_{\mathrm{a}}$.

This assertion is carefully discussed in [10]. In the tensile test, and whatever the kinematical approach

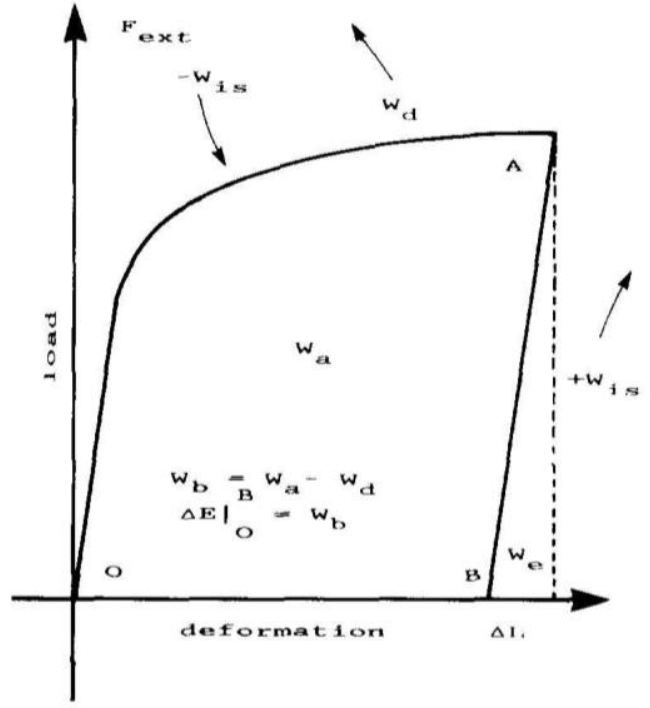

Fig. 1. Energy balance for a load-unload cycle.

used, elastic and plastic strain rate tensors can be defined and respectively noted $\dot{\boldsymbol{\epsilon}}_{\mathrm{e}}$ and $\dot{\boldsymbol{\epsilon}}_{\mathrm{p}}$ :

$\dot{\boldsymbol{\epsilon}}=\dot{\boldsymbol{\epsilon}}_{\mathrm{e}}+\dot{\boldsymbol{\epsilon}}_{\mathrm{p}}$,

where $\dot{\boldsymbol{\epsilon}}$ is the Eulerian strain rate tensor, and where:

$W_{\text {ext }}=\int_{0}^{t} \int_{\Omega(\tau)} \sigma: \dot{\boldsymbol{\epsilon}} \mathrm{d} x \mathrm{~d} \tau$

$W_{\mathrm{e}}=\int_{0}^{t} \int_{\Omega(\tau)} \sigma: \dot{\boldsymbol{\epsilon}}_{\mathrm{e}} \mathrm{d} x \mathrm{~d} \tau$,

$W_{\mathrm{a}}=\int_{0}^{t} \int_{\Omega(\tau)} \sigma: \dot{\boldsymbol{\epsilon}}_{\mathrm{p}} \mathrm{d} x \mathrm{~d} \tau$,

$\sigma$ being the Cauchy's stress tensor, $\Omega(t)$ being the geometrical domain occupied by the sample, and : symbolizing the double product of the tensor, $\left(\sigma: \epsilon=\sigma_{i j} \epsilon_{i j}\right)$.

The anelastic energy can also be decomposed into the dissipated energy $W_{\mathrm{d}}$, and a complementary part, $W_{\mathrm{b}}$, stored during the strain hardening,

$W_{\mathrm{a}}=W_{\mathrm{d}}+W_{\mathrm{b}}$.

The use of classical results descended from the Thermodynamics of Irreversible Processes, allows to define the intrinsic dissipation:

$\mathscr{D}_{1}=-p_{\mathrm{i}}-\rho \frac{\partial \psi}{\partial \alpha_{j}} \dot{\alpha}_{j}$,

where $p_{\mathrm{i}}=-\sigma: \dot{\boldsymbol{\epsilon}}$, represents the internal power per unit volume, and $\rho\left[\left(\partial \psi / \partial \alpha_{j}\right) \dot{\alpha}_{j}\right]$, the power associated 
to the set of internal state variables $\left(\alpha_{j}\right), j=1,2, \ldots, n$ as a function of the specific free energy $\psi\left(T, \alpha_{j}\right)$; ( $T=\alpha_{0}$ being the absolute temperature, and $\rho$ the mass density).

The dissipation is related to the dissipated energy by

$$
W_{\mathrm{d}}=\int_{0}^{t} \int_{\Omega(\tau)} \mathscr{D}_{1} \mathrm{~d} x \mathrm{~d} \tau
$$

It appears as heat source in the second member of the heat conduction equation:

$\rho C_{\alpha} \dot{T}+\operatorname{div} q=\mathscr{D}_{1}+r+\rho T \frac{\partial^{2} \psi}{\partial \alpha_{j} \partial T} \dot{\alpha}_{j}$,

where $C_{\alpha}$ is the heat capacity when $\left(\alpha_{j}\right) j=1, \ldots, n$ remain constant, $q$ is the heat influx, and $r$ the external heat supply.

If we assume that in the vicinity of the thermal equilibrium, the variations of the hardening parameters (i.e. the thermodynamical forces related to the hardening) $\rho \partial \psi / \partial \alpha_{j}, j=2, \ldots, n$, with the temperature are negligible, then:

$\rho C_{\alpha} \dot{\theta}-k \Delta \theta=\mathscr{D}_{1}+\rho T \frac{\partial^{2} \psi}{\partial \theta \partial \epsilon_{\mathrm{e}}}: \dot{\epsilon}_{\mathrm{e}}=\dot{w}_{\mathrm{ch}}$,

if $\theta=T-T_{\mathrm{a}},\left(T_{\mathrm{a}}\right.$ : equilibrium temperature $), r$ is constant, $q=-k$ grad $\theta$ (Fourier's law), and where $\alpha_{1}=\epsilon_{\mathrm{e}}$ is an elastic strain tensor.

In the second expression in eq. (10), near the dissipation, the isentropic term, coming from the thermoelastic effects, can be recognized. It can be related to the isentropic energy, which appears in fig. 1, during the load and the elastic unload, by the relationship:

$$
\begin{aligned}
-W_{\text {is }} & =\int_{0}^{t} \int_{\Omega(\tau)} \rho T \frac{\partial^{2} \psi}{\partial \epsilon_{\mathrm{e}} \partial T}: \dot{\epsilon}_{\mathrm{e}} \mathrm{d} x \mathrm{~d} \tau \\
& \simeq-\int_{0}^{t} \int_{\Omega(\tau)} \lambda T_{\mathrm{a}} \operatorname{tr}(\dot{\sigma}) \mathrm{d} x \mathrm{~d} \tau
\end{aligned}
$$

where $\lambda$ is the coefficient of linear thermal dilatation, $\operatorname{tr}(\square)$ symbolizing the tensor trace.

The complete balance between states $O$ and $B$ (see fig. 1) shows that the variations of internal energy $\Delta E$ of the sample corresponds to the stored energy variations

$$
\left.\Delta E\right|_{o} ^{B}=W_{\mathrm{b}}=\int_{0}^{t} \int_{\Omega(\tau)} \rho \frac{\partial \psi}{\partial \alpha_{j}} \dot{\alpha}_{j} \mathrm{~d} x \mathrm{~d} \tau, \quad j=2, \ldots, n .
$$

\section{Experimental set-up}

The large diversity of experimental approaches met in the literature, which measure the internal energy stored during the hardening, shows the difficulties of that kind of measurement. This method can be divided into two categories: the quantities of stored energy can be determined either directly, during the hardening, or indirectly, after deformation, during an appropriate cooling:

- direct approaches: between two hardening states, the stored energy is the difference between the anelastic energy and the dissipated work $[6,14,15]$;

- indirect approaches: the stored energy is determined by comparing the thermal behaviour of a hardened sample with a cooled one, during a cooling process [16].

The wide range of results, obtained on copper (see fig. 2), for instance, leads us to suggesting the following experimental set-ups. The first one, is a microcalorimeter, adapted to tensile testing; the second one uses the infrared techniques developed in the Laboratoire de Mécanique et d'Acoustique de Marseille, France. It must be noted that both direct approaches are independent and complementary.

\subsection{Microcalorimetry of tensile test}

The originality of such a device is that the microcalorimeter is directly placed on the crosshead of the testing machine, and the sample passing through the

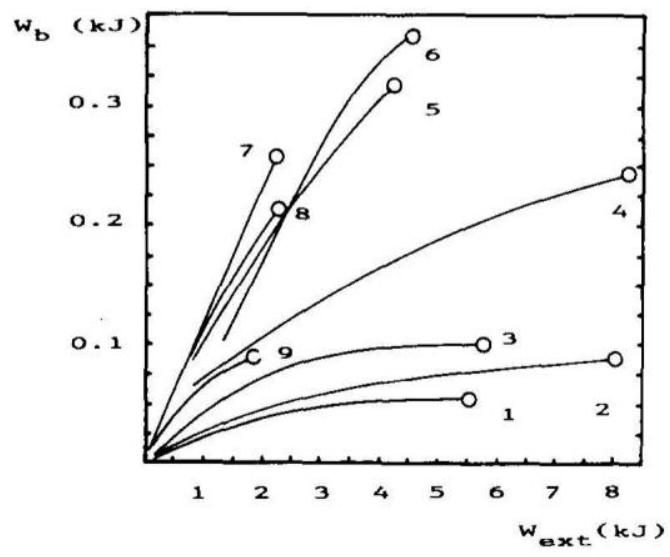

Fig. 2. Stored energy dispersion in case of copper. Experimental results synthesis extracted from [16]. (1.2) L.M. Clarebrough, (3) S. Sato, (4) V.I. Khotkevich, (5) S.D. Hertsariken, (6) G.I. Taylor, H. Quinney, (7) T. Suzuki, (8) M.M. Degtiarev, (9) R.O. Williams. 


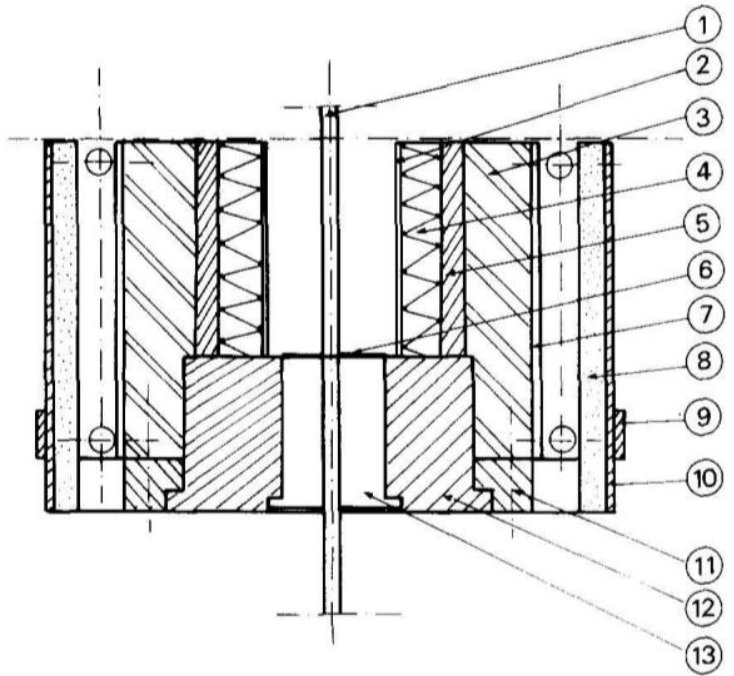

Fig. 3. Microcalorimeter: (1) sample, (2) internal cell cylinder, (3) Aluminium block, (4) thermocouples, (5) external cell cylinder, (6) anti radiation screen, (7),..,,(13) thermal resistances.

cell, (fig. 3). The main advantage is that the thermocouples are not in contact with one another.

In general, with such a device, the measurements are realized between two thermal equilibrium states. Here, one of the objectives was to realize a microcalorimeter able to evaluate continuously the heat evolved during the test. In comparison with Calvet's classical calorimeter, our device possesses only one cell with 1200 thermocouples, instead of two differential cells with 100 to 400 thermocouples (the sensitivity of the classical microcalorimeter can reach $5 \times 10^{-6 \circ} \mathrm{C}$ between both cells). Here, the heat losses due to the sample imply a maximal sensitivity of $10^{-3 \circ} \mathrm{C}$.

To obtain a good signal-to-noise ratio, the calibration of the thermoregulation must be carefully carried out. The test room must be completely closed. The air is mixed by six electric fans and climatized by two heat sources. The efficiency of the thermoregulation comes afterwards from the series of thermal resistances placed between the test room air and the cell. This one is made by two coaxial copper cylinders between which the thermocouples are regularly placed.

To relate the signal given by the thermopile to the heat evolved in the cell, several hypotheses are made.

It is supposed that in the vicinity of thermal equilibrium, the heat transfers, between the sample and the internal cell cylinder, between the internal and external cylinders, between the sample or the cell and the surroundings, by radiation, convection or conduction, are well modelled by linear laws in temperature. Therefore, if $T_{\mathrm{i}}, T_{\mathrm{e}}$, are respectively the mean temperature of the internal and the external cylinders, the signals delivered by the thermocouples is proportional to the thermal disequilibrium

$s=G\left(T_{\mathrm{i}}-T_{\mathrm{e}}\right)$, where $G$ is a constant.

Integrating the heat conduction equation into the deformed volume of the sample, into the internal and external cell cylinder yields the following differential system:

$\left\{\begin{array}{l}\mu_{1} \dot{T}_{\mathrm{m}}+H_{1}\left(T_{\mathrm{m}}-T_{\mathrm{i}}\right)+H_{2}\left(T_{\mathrm{m}}-T_{\mathrm{a}}\right)=\dot{Q} \\ \mu_{2} \dot{T}_{\mathrm{i}}+H_{3}\left(T_{\mathrm{i}}-T_{\mathrm{e}}\right)-H_{1}\left(T_{\mathrm{m}}-T_{\mathrm{i}}\right)=0, \\ \mu_{3} \dot{T}_{\mathrm{e}}+H_{4}\left(T_{\mathrm{e}}-T_{\mathrm{a}}\right)-H_{3}\left(T_{\mathrm{i}}-T_{\mathrm{e}}\right)=0,\end{array}\right.$

where $T_{\mathrm{m}}$ is the characteristic temperature of the sample and where the test room temperature is supposed to be constant; $\mu_{1}, \mu_{2}, \mu_{3}$ are respectively the heat capacities of the sample, the internal and external cell cylinders; $H_{1}, H_{2}, H_{3}, H_{4}$ characterize the heat transfers between the sample and the cell inside, the sample and the surroundings, the internal cylinder and the external one, the external cylinder and the surroundings; and $\dot{Q}=\dot{W}_{\mathrm{d}}-\dot{W}_{\text {is }}$ represents the mechanical heat source.

The relationship between the evolved heat $Q$ and the signal $s$ can be deduced from eqs. (13) and (14):

$$
\begin{gathered}
t_{\mathrm{a}} \dot{s}(t)+s(t)+\int_{0}^{t}\left[\frac{1}{t_{\mathrm{b}}}+\frac{1}{\tau_{1}}\left(1-\frac{t_{\mathrm{a}}}{\tau_{2}}\right)\right. \\
\left.\times \exp \left(-\frac{t-\tau}{\tau_{2}}\right)\right] s(\tau) \mathrm{d} \tau=K Q(t),
\end{gathered}
$$

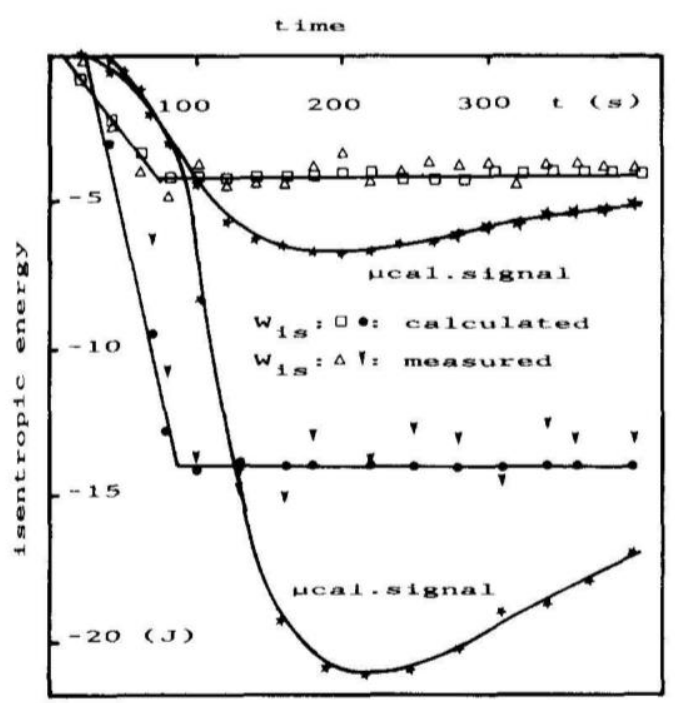

Fig. 4. Calibration control of the microcalorimetric device. 
where $t_{\mathrm{a}}, t_{\mathrm{b}}, \tau_{1}, \tau_{2}$ and $K$ are constants which can be experimentally evaluated [17]. A validity control, and a calibration of the device is made using several thermoelastic effects: monotonic and cyclic elastic solicitations are used. Then, the measurement of the isentropic energy is compared with its theoretically calculated value (linear and isotropic behaviour law), (cf. fig. 4).

\subsection{Infrared thermography}

The second approach uses infrared techniques. The numerization system developed in the Laboratoire de Mécanique et d'Acoustique de Marseille by Nayroles $e t$ $a l$. is adapted to the energy balances study [18-21].

To illustrate the performances of such an apparatus, we can mention some data. The observation zone, of the camera, is in our experiments, a rectangle of $15 \mathrm{~mm} \times 25$ $\mathrm{mm}$. Each thermal picture represents a matrix of $128 \times$ 180 points. The recording time, on computer, is around $0.6 \mathrm{~s}$ and the information recorded during a test represents a data array of 27 mega bytes. The resolution is around $0.13 \mathrm{~mm}$ for a maximal sensitivity of $2.7 \times$ $10^{-3 \circ} \mathrm{C}$.

The temperature maps are used to determine, by the interposition of the heat conduction equation, the heat locally evolved.

The main difficulty in estimating the differential operator applied to the temperature, comes from the thermal noise, which is essentially due to the parasite reflexions.

To estimate the first expression in eq. (10), the chosen method is the following:

$\frac{\partial \theta}{\partial t}+u \operatorname{grad} \theta-\frac{k}{\rho C_{\alpha}} \Delta \theta=\frac{1}{\rho C_{\alpha}}\left(\mathscr{D}_{1}-\dot{w}_{\text {is }}\right)$,

where $u$ is the velocity vector.

In our experiments, the convection term is negligible (good thermal diffusivity of the studied materials, and very short strain rate). The local equation is integrated onto a little surface $S$ of the observation zone, in order to eliminate the Laplacian term, which is a big noise amplifier

$$
\frac{\partial \bar{\theta}}{\partial t}-\frac{k}{\rho C_{\alpha}} \int_{\partial S} \frac{\partial \theta}{\partial n} \mathrm{~d}(\partial S)=\frac{1}{\rho C_{\alpha}}\left(\overline{\mathscr{D}}_{1}-\overline{\dot{w}}_{\text {is }}\right),
$$

the bar symbolizing the average on $S$.

Then, we assume, once more, that in the vicinity of thermal equilibrium, the heat flux by radiation and conduction (no convection, experiments are made under

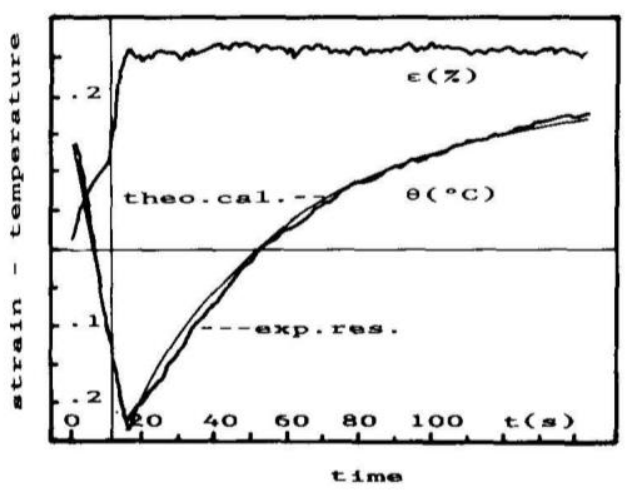

Fig. 5. Calibration control of the infrared device.

a primary vacuum), can be modelled by a linear law in temperature:

$\frac{\partial \bar{\theta}}{\partial t}+\frac{\bar{\theta}}{\tau_{\text {th }}}=\frac{1}{\rho C_{\alpha}}\left(\overline{\mathscr{D}}_{1}-\overline{\dot{w}}_{\text {is }}\right)$

the time constant $\tau_{\mathrm{th}}$ characterizes the local heat losses.

To illustrate the incidence of the thermal noise upon the heat source determination, we point out the fact that without efficient numerical filtering, the heat source fluctuations due to the Laplacian term, are around $250^{\circ} \mathrm{C} / \mathrm{s}$ when the order of magnitude of the dissipation is around $10^{-2}-10^{-10} \mathrm{C} / \mathrm{s}$.

The validity of the hypothesis of the linearity of losses is carefully controlled, by heating the sample (Joule's effects), by studying the cooling or reheating of the sample caused by the thermoelastic couplings, and by calculating, in the center of the sample, the analytical solution of one thermal problem associated with boundary conditions which are close to the experimental ones, (cf. fig. 5).

\section{Experimental results}

Tests are made on duralumin (AU4G), carbon steel (XC38) and stainless steel (A316L). We suppose homogeneous tensile tests; therefore, the deformation is limited to $10 \%$ in order to neglect any local striction or damage. Young's modulus remains constant during the different deformation processes. The load and deformation signals allow us to determine the strain and stress states. They are used to evaluate the plastic work $W_{\mathrm{a}}$ and the isentropic energy $W_{\text {is }}$.

Both approaches lead to results in good agreement. They are very similar at the beginning of the hardening, the dispersion increasing with the processes (figs. 6 and 


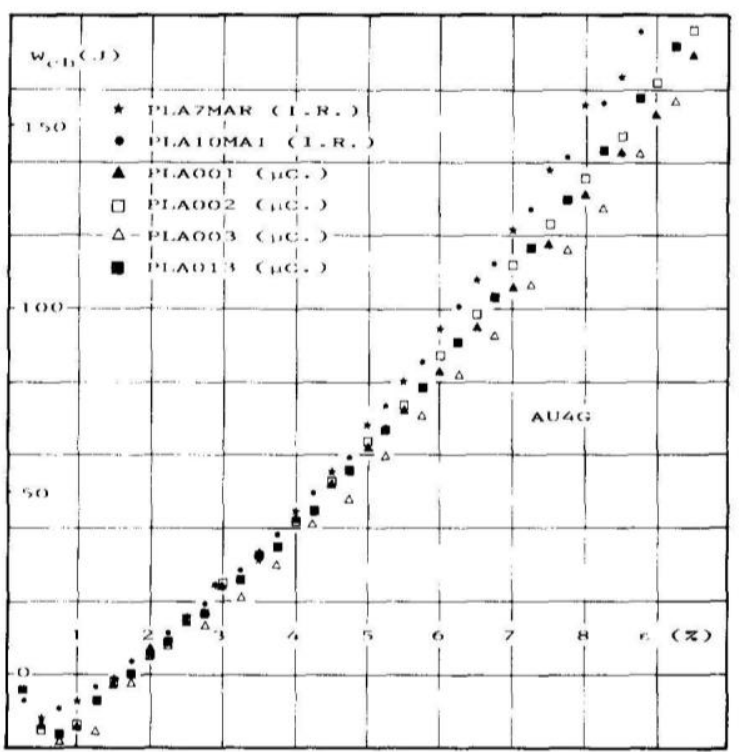

Fig. 6. Dispersion of evolved heat measured by microcalorimetric $(\mu \mathrm{c})$ and infrared (IR) experimental approaches. Supercooling duralumin. Equivalent volume: $8.4 \times 10^{-6} \mathrm{~m}^{3}$.

7). The relative errors, concerning the heat quantity measurements begin at 3 or $4 \%$ and do not exceed $15 \%$.

The experiments made on the three materials mentioned above have shown that:

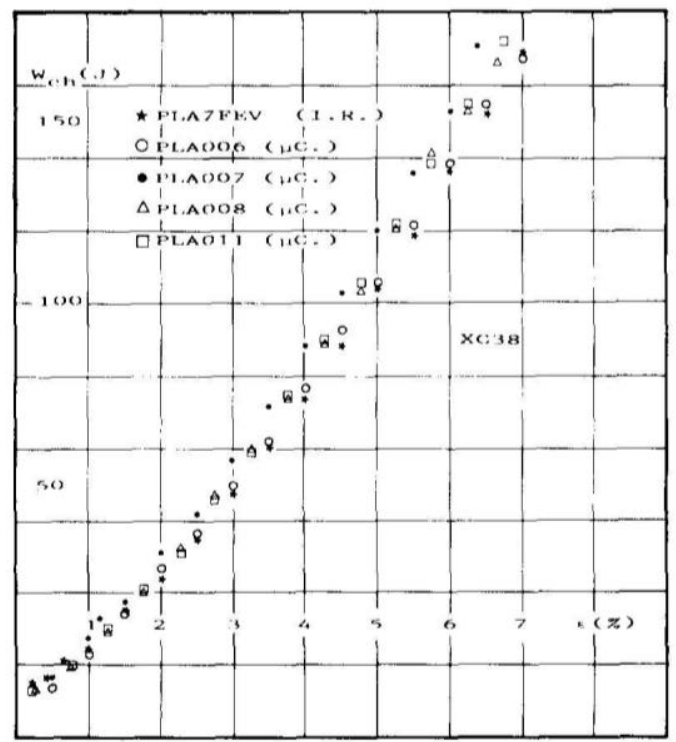

Fig. 7. Dispersion of evolved heat by $(\mu c)$ and (IR) approaches. Age-hardening carbon steel. Equivalent volume: 8.4 $\times 10^{-6} \mathrm{~m}^{3}$

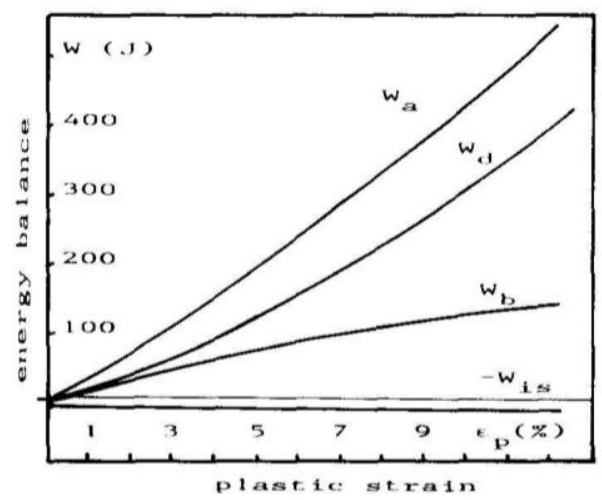

Fig. 8. Energy balance in case of carbon steel (equivalent volume $8.4 \times 10^{-6} \mathrm{~m}^{3}$ ).

- the stored energy evolution is limited, during a monotonic strain hardening, (figs. 8 and 9). That implies a decrease of the stored energy ratio $\left(W_{\mathrm{b}} / W_{\mathrm{a}}\right)$ which reaches $50-60 \%$ at the beginning of the hardening;

- in the first approximation, the stored energy is a linear function of the applied stress, as soon as plastic deformation appears (fig. 10).

If the decrease of the stored energy ratio is now a classical result [4], the percentages obtained here are more important than the ones often met in the literature; generally $W_{\mathrm{b}} / W_{\mathrm{a}}$ do not exceed $15 \%$, what explains the classical attitude in behaviour law modelling of neglecting the stored energy of cold work, [22]:

$\rho \frac{\partial \psi}{\partial \alpha_{j}} \dot{\alpha}_{j} \ll \sigma: \dot{\boldsymbol{\epsilon}}_{\mathrm{p}}, \quad j=2, \ldots, n$.

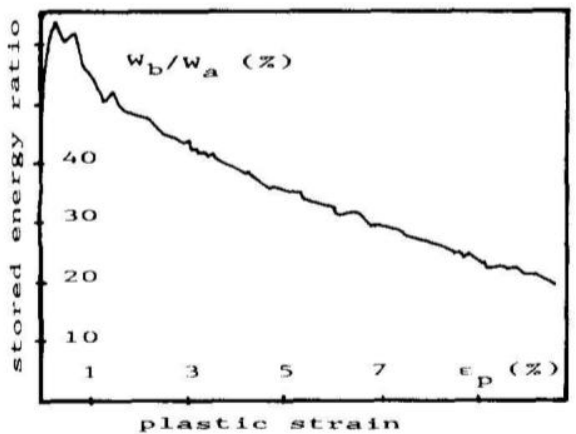

Fig. 9. Decrease of the stored energy ratio (supercooling duralumin). 


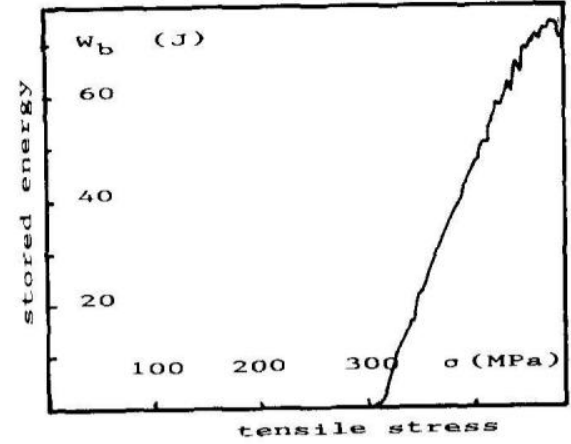

Fig. 10. Linear evolution of the stored energy as a function of stress (supercooling duralumin).

In our case, this approximation cannot be more justified, especially at the beginning of strain hardening where:

$\rho \frac{\partial \psi}{\partial \alpha_{j}} \dot{\boldsymbol{\alpha}}_{j} \simeq \mathscr{D}_{1} \simeq \frac{1}{2} \boldsymbol{\sigma}: \dot{\boldsymbol{\epsilon}}_{\mathrm{p}}, \quad j=2, \ldots, n$.

The evolution of the stored energy is also an original result. On pure metals, like copper or aluminium, the evolutions of the stored energy as a function of stress, are often modelled by quadratic curves [23] or cubic curves [14].

\section{Validity control of thermomechanical behaviour laws and new models propositions}

Even if some behaviour laws are able to qualitatively predict some of the observed energy phenomena, we would still hold it preferable to begin the examination of thermomechanical models using the laws previously mentioned. We could have taken, for instance, the nonlinear kinematical hardening model which assures qualitatively the decrease of the stored energy ratio [24]. The chosen models are sufficient to describe monotonic hardening phenomena and are the basis of all models founded on isotropic or kinematical hardening. The validity of both laws is determined by comparing their predictions, from an energy point of view, with experimental observations during tensile tests.

In case of an isotropic and/or kinematical model the intrinsic dissipation can be written:

$\mathscr{D}_{1}=\sigma: \dot{\epsilon}_{\mathrm{p}}-X: \dot{\alpha}-R \dot{p}$

where $\sigma$ is Cauchy's stress tensor, $\dot{\epsilon}_{\mathrm{p}}$ the plastic strain rate, $(X, \alpha)$ and $(R, p)$ the couples (thermodynamical force, state variable) respectively associated with the kinematical and isotropical hardening.

When the elastic domain is defined by Von Mises's criterion (cf. fig. 11), and if the generalized normality hypothesis is presumed [7], the dissipation can be rewritten:

$\mathscr{D}_{1}=R_{0} \dot{\epsilon}_{\mathrm{pII}}$,

where $\dot{\alpha}=\dot{\epsilon}_{\mathrm{p}}, \dot{\mathrm{p}}=\dot{\epsilon}_{\mathrm{pII}},(\square)_{\mathrm{II}}$ symbolizing the second invariant of the tensor.

In the case of monotonic, homogeneous tensile tests

$\mathscr{D}_{1}=\sigma_{e} \dot{\varepsilon}_{p_{3}}$

where $\sigma_{e}$ is the yield stress and $\dot{\epsilon}_{p_{3}}$ is the tensile strain rate, and where index 3 symbolizes the tensile direction.

The stored energy by volume rate is by definition, the difference between the anelastic energy rate and the dissipated power:

$\dot{w}_{\mathrm{b}}^{\text {thoo }}=\sigma: \dot{e}_{\mathrm{p}}-\mathscr{D}_{1}=\left(\sigma_{\mathrm{II}}-R_{0}\right) \dot{\epsilon}_{\mathrm{pII}} \geqslant 0$.

In the case of monotonic tensile tests:

$\dot{w}_{\mathrm{b}}^{\text {thoo }}=\left(\sigma_{\mathrm{t}}-\sigma_{\mathrm{e}}\right) \dot{\epsilon}_{\mathrm{p}_{3}}$,

where $\sigma_{t}$ is the tensile stress. We stress the fact that, during such tests, if the plastic strain rate $\dot{\epsilon}_{\mathrm{p}_{3}}$ remains approximately constant, $\left(\ddot{\epsilon}_{\mathrm{p}} \simeq 0\right)$, eq. (25) implies the indefinite increasing of the stored energy:

$\ddot{w}_{\mathrm{b}}^{\text {theo }} \simeq \dot{\sigma}_{\mathrm{c}} \dot{\mathrm{e}}_{\mathrm{p}_{3}} \geqslant 0$.

This result is not similar to experimental observations. If the stored energy increases at the beginning of the hardening, the material asymptotically tends to a saturation state

$\ddot{w}_{\mathrm{b}}^{\exp } \leqslant 0$.

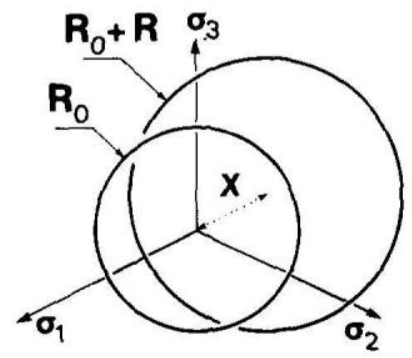

Fig. 11. Von Mises's criterion: $f(\sigma, R, X)=(s-X)_{\mathrm{II}}-R-$ $R_{0}$. 


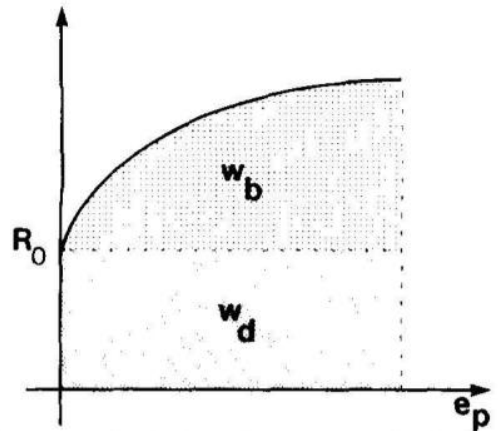

Fig. 12. Stored and dissipated energy evolutions predicted by Prandtl-Reuss's and Prager's behaviour laws.

The confrontation between theory and experiment is more significant if we consider the stored energy ratio $\mathscr{F}$ :

$\mathscr{F}=\frac{\int_{0}^{t} \int_{\Omega(\tau)} \dot{\mathcal{W}}_{\mathrm{b}} \mathrm{d} x \mathrm{~d} \tau}{\int_{0}^{t} \int_{\Omega(\tau)} \sigma: \dot{\epsilon}_{\mathrm{p}} \mathrm{d} x \mathrm{~d} \tau}=\frac{W_{\mathrm{b}}}{W_{\mathrm{a}}}$.

Experimentally, the decreasing of the stored energy ratio as a function of the cumulated plastic strain $e_{\mathrm{p}}$ is shown, $\left(e_{\mathrm{p}}=\int_{0}^{t} \dot{\epsilon}_{\mathrm{pII}} \mathrm{d} \tau\right)$, when the models predict an increasing evolution, (fig. 12).

In order to modify such models, we abandon the hypothesis that the hardening parameters can be directly identified with the thermodynamic forces, and we will suppose that the first ones are dependent on the second ones.

\subsection{Proposition of isotropic model}

In the case of isotropic hardening, with classical notations, the Helmholtz's free energy per unit volume can be written

$\rho \psi\left(\theta, \epsilon_{\mathrm{e}}, p\right)=\frac{1}{2} \Lambda(\theta): \epsilon_{\mathrm{e}}: \epsilon_{\mathrm{e}}+\psi_{\mathrm{h}}(\theta, p)$.

We will suppose that the (small) temperature variations due to deformation do not modify the hardening state of material:

$\frac{\partial \psi_{\mathrm{h}}}{\partial \theta}=0 \quad$ and $\quad w_{\mathrm{b}}=\psi_{\mathrm{h}}$.

The thermodynamical forces associated to the state variables $\epsilon_{\mathrm{e}}$ and $p$ are respectively $\sigma$ and $-z$ defined by:

$\sigma=\rho \frac{\partial \psi}{\partial \epsilon_{\mathrm{e}}}$, $z=\rho \frac{\partial \psi}{\partial p}=\frac{\mathrm{d} \psi_{\mathrm{h}}}{\mathrm{d} p}$.

Note that we have kept $p$ as the symbol for the state variable associated with the isotropic hardening, but now $p$ is a priori different from the cumulated plastic strain $e_{\mathrm{p}}$.

Von Mises's criterion is a function of thermodynamic forces:

$f(\sigma, z)=(s)_{\mathrm{II}}-R(z)-R_{0}$,

where $(s)_{\mathrm{II}}$ is the second invariant of the stresses deviator. The generalized normality hypothesis leads to:

$\dot{\epsilon}_{\mathrm{p}}=\lambda \frac{\partial f}{\partial \boldsymbol{\sigma}}=\lambda \frac{s}{(s)_{\mathrm{II}}}$,

$\lambda=\left(\dot{\epsilon}_{\mathrm{p}}\right)_{\mathrm{II}}$,

$\dot{p}=-\lambda \frac{\partial f}{\partial z}=\frac{\mathrm{d} R}{\mathrm{~d} z}\left(\dot{\epsilon}_{\mathrm{p}}\right)_{\mathrm{II}}$.

The intrinsic dissipation can be written:

$\mathscr{D}_{1}=\sigma: \dot{\epsilon}_{\mathrm{p}}-z \dot{p}=\sigma: \dot{\epsilon}_{\mathrm{p}}-\dot{\psi}_{\mathrm{h}}$.

Then, the one-to-one map between the hardening parameter $R$ and the thermodynamic force $-z$ and the evolution equation of the state variable $p$ can be deduced from the experimental results.

First, one approximates the tensile hardening curve by an exponential branch:

$\sigma_{\mathrm{t}}\left(e_{\mathrm{p}}\right)=\sigma_{\mathrm{e}}+\sigma_{\mathrm{s}}\left[1-\exp \left(-a e_{\mathrm{p}}\right)\right]$,

where $\sigma_{e}$ is the yield stress, $\sigma_{\mathrm{s}}$ and $a$ are constants.

Secondly, we have seen that the stored energy evolution as a function of stress could be well approached by linear law:

$\psi_{\mathrm{h}}\left(e_{\mathrm{p}}\right)=\frac{1}{b} R\left(e_{\mathrm{p}}\right)$,

where $b$ is a constant and $R\left(e_{\mathrm{p}}\right)=\left(\frac{2}{3}\right)^{1 / 2}\left(\sigma_{\mathrm{t}}\left(e_{\mathrm{p}}\right)-\sigma_{\mathrm{e}}\right)$.

Then, with eqs. (32), (36), (38), (39), we get an evolution equation for $p$ :

$\dot{p}=(a+b)\left(p_{\mathrm{s}}-p\right)\left(\dot{\epsilon}_{\mathrm{p}}\right)_{\mathrm{II}}$,

where $p_{\mathrm{s}}$ is a constant.

The $z(R)$ map is:

$z=\frac{\mathrm{d} \psi_{\mathrm{h}}}{\mathrm{d} p}=\frac{R_{0} f_{0}}{(a+b) p_{\mathrm{s}}}\left(1-\frac{R}{R_{\mathrm{s}}}\right)^{-b / a}$,

where $R_{0}=\left(\frac{2}{3}\right)^{1 / 2} \sigma_{\mathrm{e}}$ and $R_{\mathrm{s}}=\left(\frac{2}{3}\right)^{1 / 2} \sigma_{\mathrm{s}}$, and where $f_{0}$ is 


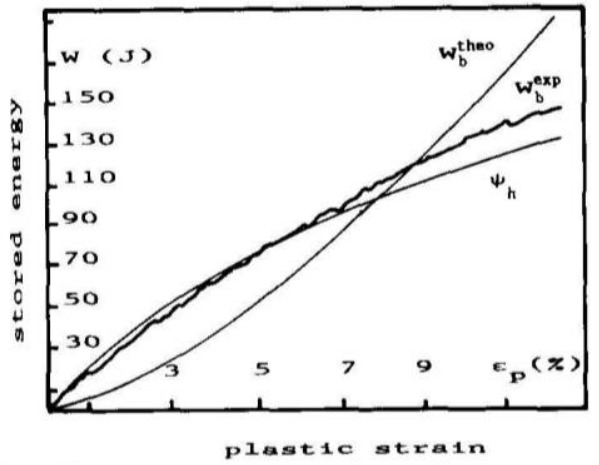

Fig. 13. Calculated and measured stored energy evolutions. Equivalent volume: $8.4 \times 10^{-6} \mathrm{~m}^{3}$. Supercooling duralumin.

a constant. And finally, the stored energy evolution becomes: (figs. 13, 14, 15)

$\psi_{\mathrm{h}}\left(e_{\mathrm{p}}\right)=\psi_{\mathrm{s}}\left[1-\exp \left(-a e_{\mathrm{p}}\right)\right]$,

where

$\psi_{\mathrm{s}}=\left(\frac{2}{3}\right)^{1 / 2} \frac{\sigma_{\mathrm{e}}}{a} f_{0}$.

The $p_{\mathrm{s}}$ constant is an arbitrary constant which symbolizes the asymptotic value reached by the hardening state variable * when the saturated material behaviour tends to perfect plastic behaviour $\left(\dot{\sigma}_{t} \simeq 0\right)$. The $f_{0}$ constant is the initial fraction of the stored energy ratio:

$\lim _{e_{\mathrm{p}} \rightarrow 0} \mathscr{F}=\lim _{e_{\mathrm{p}} \rightarrow 0} \frac{\psi_{\mathrm{h}}\left(e_{\mathrm{p}}\right)}{w_{\mathrm{a}}\left(e_{\mathrm{p}}\right)}=f_{0}$.

The couple $(-z, p)$ associated with the hardening state can be completely defined with the mechanical parameters and with $f_{0}$ :

$z=\left(\frac{2}{3}\right)^{1 / 2} \cdot \frac{f_{0}}{(a+b) p_{\mathrm{s}}} \cdot \sigma_{\mathrm{e}}\left(1-\frac{\sigma_{\mathrm{t}}-\sigma_{\mathrm{e}}}{\sigma_{\mathrm{s}}}\right)^{-b / a}$,

$p=p_{\mathrm{s}} \cdot\left\{1-\exp \left[-(a+b) e_{\mathrm{p}}\right]\right\}$.

\subsection{A suggested kinematical model}

In the case of kinematical hardening, the same approach is followed. The Helmholtz free energy is written:

$\rho \psi\left(\theta, \epsilon_{\mathrm{e}}, \alpha\right)=\frac{1}{2} \Lambda(\theta): \epsilon_{\mathrm{e}}: \epsilon_{\mathrm{e}}+\psi_{\mathrm{h}}(\alpha)$,

- For instance, the dislocation density.

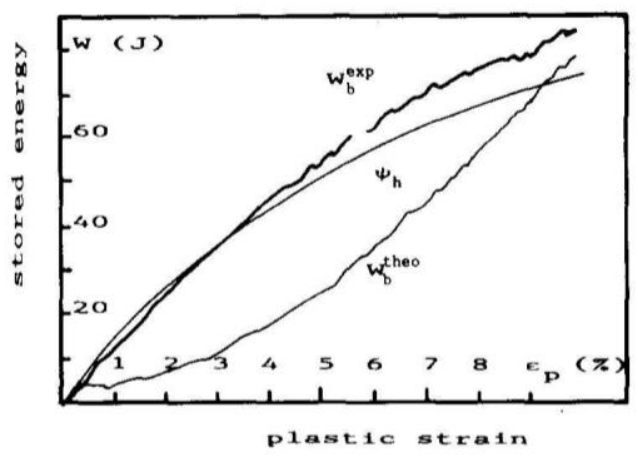

Fig. 14. Stored energy evolutions. Age-hardening carbon steel.

where

$\sigma=\rho \frac{\partial \psi}{\partial \epsilon_{\mathrm{e}}}$,

$Z=\rho \frac{\partial \psi}{\partial \alpha}=\frac{d \psi_{h}}{d \alpha}$

Von Mises's criterion is once more chosen:

$f(\sigma, Z)=\left(s-X(Z)_{\mathrm{II}}\right)-R_{0}$.

The one-to-one map between $X$ and $Z$ is supposed; and the generalized normality hypothesis leads to:

$$
\begin{aligned}
& \dot{\boldsymbol{\epsilon}}_{\mathrm{p}}=\lambda \frac{\partial f}{\partial \sigma}=\lambda \frac{s-X}{(s-X)_{\mathrm{II}}}, \\
& \lambda=\left(\dot{\epsilon}_{\mathrm{p}}\right)_{\mathrm{II}}, \\
& \dot{\alpha}=-\lambda \frac{\partial f}{\partial Z}=\frac{\mathrm{d} X}{\mathrm{~d} Z}: \dot{\epsilon}_{\mathrm{p}} .
\end{aligned}
$$

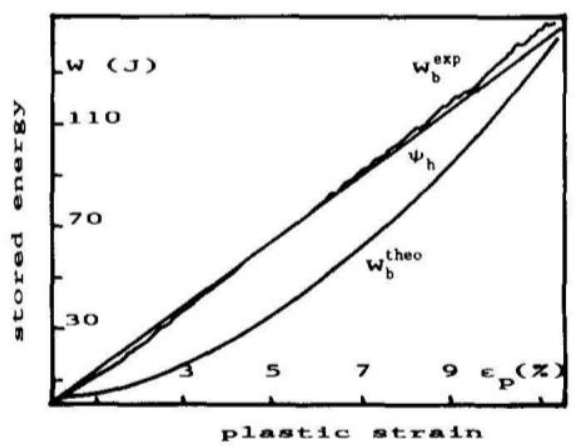

Fig. 15. Stored energy evolutions. Age-hardening stainless steel. 
The form of the experimental results leads to the choice of an evolution equation for $\alpha$ analogous to the one for $p$ :

$\dot{\alpha}=c\left(\alpha_{s}\right)_{\mathrm{II}}\left(1-\frac{\alpha}{\left(\alpha_{\mathrm{s}}\right)_{\mathrm{II}}} \otimes n\right): \dot{e}_{\mathrm{p}}$

where

$n=\frac{s-X}{(s-X)_{\mathrm{II}}}$.

The $\left(\alpha_{s}\right)_{11}$ constant is exactly like $p_{\mathrm{s}}$ in the isotropic case; an arbitrary constant which could be related to the microscopic parameters like dislocation density, dislocation cell size, etc.

The thermodynamical force $X$ is supposed to be a deviatory tensor, and to have the representative matrix:

$X=\left[\begin{array}{ccc}-X / 2 & 0 & 0 \\ 0 & -X / 2 & 0 \\ 0 & 0 & X\end{array}\right]$,

relatively to an orthonormal frame of reference $\left(x_{1}, x_{2}, x_{3}\right)$ where $O x_{3}$ is the loading direction.

The modelling of the tensile hardening curve by an exponential branch leads to:

$X=X_{s}\left[1-\exp -\left(a e_{\mathrm{p}}\right)\right]$

where

$X_{\mathrm{s}}=\left[\begin{array}{ccc}-X_{\mathrm{s}_{3} / 2} & 0 & 0 \\ 0 & -X_{\mathrm{s}_{3} / 2} & 0 \\ 0 & 0 & X_{\mathrm{s}_{3}}\end{array}\right], \quad X_{\mathrm{s}_{3}}$ being constant.

The linear evolution of the stored energy with stress becomes:

$\psi_{\mathrm{h}}\left(e_{\mathrm{p}}\right)=\frac{1}{b}(X)_{\mathrm{II}}\left(e_{\mathrm{p}}\right)$.

The integration of eq. (53), in case of $\alpha=0$ for a virgin sample, leads to:

$\alpha=\alpha_{\mathrm{s}}\left[1-\exp \left(-c e_{\mathrm{p}}\right)\right]$,

where

$c=a+b \quad$ and $\quad \alpha_{\mathrm{s}}=\left[\begin{array}{ccc}-\alpha_{\mathrm{s}_{3} / 2} & 0 & 0 \\ 0 & -\alpha_{\mathrm{s}_{3} / 2} & 0 \\ 0 & 0 & \alpha_{\mathrm{s}_{3}}\end{array}\right]$.
The stored energy evolution can be rewritten as:

$\psi_{\mathrm{h}}\left(e_{\mathrm{p}}\right)=\psi_{\mathrm{s}}\left[1-\exp \left(-a e_{\mathrm{p}}\right)\right]$,

where

$\psi_{\mathrm{s}}=\left(\frac{2}{3}\right)^{1 / 2} \frac{\sigma_{\mathrm{e}}}{a} f_{0}$

(see figs. 13, 14, and 15).

\section{Conclusions}

The experimental study of the energy balance is an important supplementary asset in determining the state variables and thermodynamical forces associated with the hardening modelling.

We have shown that the effect of knowledge of the energy phenomena on the determination of behaviour laws seem to be fundamental even if the associated thermal effects are negligible. For instance, we have observed, at the beginning of strain hardening, that the stored energy quantities can be as important as the dissipated ones, and cannot be neglected.

From a theoretical point of view, we have shown that our observations cannot be predicted by PrandtlReuss or Prager's behavioural laws. Both models are transformed, assuming that the introduction of hardening parameters into Von Mises's criterion depend upon the thermodynamical forces. Taking into account the stored energy evolution enables one to obtain evolution equations which are similar to the ones introduced in the non-linear kinematical model, (well adapted to describe cyclic hardening effects).

\section{References}

[1] A. Chrysochoos, O. Maisonneuve and G. Martin, Dissipative and non dissipative phenomena during an elastoplastic deformation process, in: Folker H. Wittman (A.A. Balkema, 1987), eds., Trans. 9th Int. Conf. au SMIRT, Vol. L, pp. 357-366.

[2] Lord Kelvin, On the thermoelastic and thermo-magnetic properties of matter, Trans. Roy. Soc. Edimb. 20, 161 (1853) 57-77.

[3] G.I. Taylor and H. Quinney, The latent energy remaining in a metal after cold working, Proc. Roy. Soc. London, 143A (1934) 307-326.

[4] H.D. Bui, Dissipation d'énergie dans une déformation plastique, Cah. Fran. Rhéo. 1 (1965) 15-19.

[5] J. Mandel, Energie élastique et travail dissipé dans les modèles, Cah. Fran. Rhéo. 1 (1965) 9-13. 
[6] E. Krempl, Inelastic work and thermomechanical coupling in viscoplasticity, in: A. Sawzuk and G. Bianchi, eds., Plasticity Today (Elsevier Applied Science Publishers, Amsterdam, 1985) pp. 247-257.

[7] B. Halphen and Q.S. Nguyen, Sur les matériaux standards généralisés, J. de Méc. Appl. 14, 1 (1975) 39-63.

[8] J. Bataille and J. Kestin, L'interprétation physique de la thermodynamique rationelle, J. de Mec., 14, 2 (1975) 365-384.

[9] G. Lebon and Ph. Mathieu, Etude comparée de diverses théories de thermodynamique du non équilibre, J. Entropie 100 (1981) 75-85.

[10] A. Chrysochoos, Bilan énergétique en élastoplasticité grandes déformations, J. Theo. Appl. Mech. 4, 5 (1985) 589-614.

[11] A.E. Green and P.M. Naghdi, A general theory of an elastic-plastic continuum, Arch. Rat. Mech. Ana. 18 (1965) 251-281.

[12] E.H. Lee, Elastic-plastic deformation at finite strains, J. App. Mech. 03 (1969) 1-6.

[13] S. Nemat-Nasser, Decomposition of strain measures and their rates in finite deformation elastoplasticity, Int. J. Solids Structures 15 (1979) 155-166.

[14] D. Rönnpagel and Ch. Schwink, Measurement of the stored energy of copper single crystals by means of a new deformation calorimetry method, Acta Meta. 26 (1978) 319-331.

[15] R.O. Williams, The stored energy in deformed copper: the effect of grain size and silver content, Acta Meta. 9 (1961) 949-957.
[16] M.B. Bever, D.L. Holt and A.L. Titchener, The Stored Energy of Cold Work, Progress in Materials Science 17 (Pergamon, New York, 1973).

[17] A. Chrysochoos, Dissipation et blocage d'énergie lors d'un écrouissage en traction simple, Thèse d'Etat, Montpellier (1987).

[18] B. Nayroles, R. Bouc, H. Caumon and J.C. Chezeaux, Téléthermographie infrarouge et mécanique des structures, Int. J. Engng. Sci. 19 (1981) 929-947.

[19] J.C. Chezeaux, Contrôleur d'acquisition numérique d'image thermique, Note interne L.M.A. 1200, CNRS (1984).

[20] P. Bremond, Développement d'une instrumentation infra-rouge pour l'étude des structures mécaniques, Thèse D.I., Marseille (1982).

[21] R. Bouc and B. Nayroles, Méthodes et résultats en thermographie I.R. des Solides, J. de Mec. Theo. Appl. 4 (1985) 27-58.

[22] J. Lemaitre and J.L. Chaboche, Mécanique des matériaux solides, (Dunod, Paris, 1985) pp. 39-70.

[23] J.E. Bailey and P.B. Hirsch, Phil. Mag. 5 (1960) 485.

[24] J.L. Chaboche, Sur l'utilisation des variables d'état interne pour la description du comportement viscoplastique et de la rupture par endommagement, in: French-Polish Symp., Non linear Problems in Mechanics (PWN, Warsaw, 1980) pp. 137-159.

[25] A. Chrysochoos, O. Maisonneuve, C.R. Acad. Sc. Paris, t. 300, Série II, no. 20 (1985) 985-990. 